Location Oahu to $20 \%$ i $259^{\circ} 30^{\prime} \omega$

\begin{tabular}{ll}
\hline Observer Date 25 Jawuary, $1961^{\prime}$ Time $1005^{-}$to $1425^{\prime}$ \\
Weather
\end{tabular} $65 \mathrm{miles}$

SPECIES

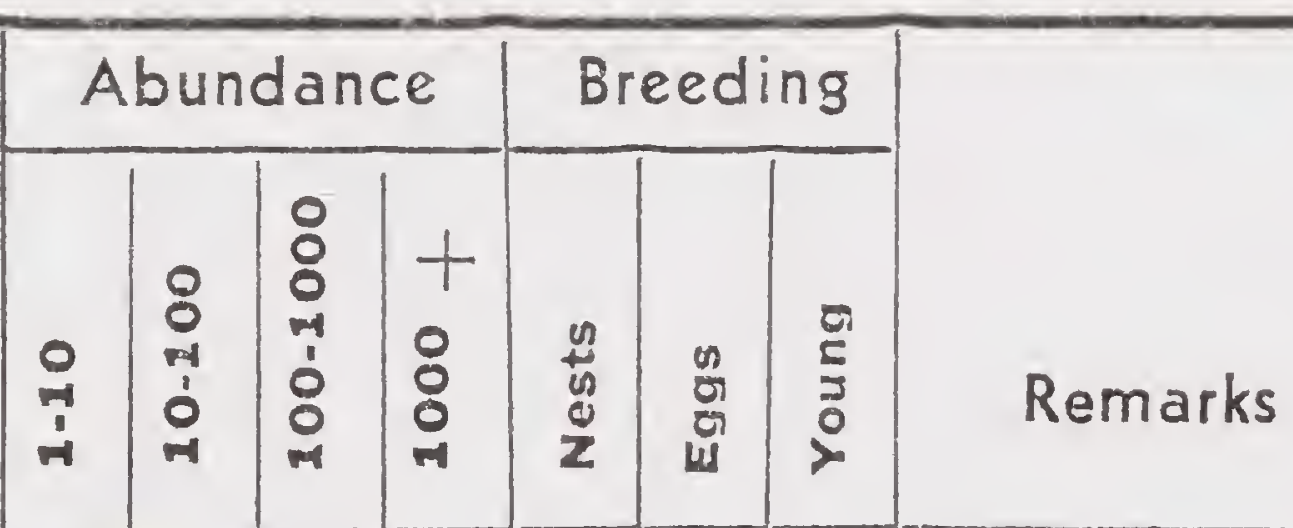

Laysan Albatross

Black-footed Albatross

Wedge-tailed Shearwater

Christmas 1. Shearwater

Audubon's Shearwater

Bonin 1. Petrel

Phoenix 1. Petrel

Bulwer's Petrel

Sooty Petrel

Red-tailed Tropicbird

White-tailed Tropicbird

Masked Booby

Brown Booby

Red-footed Booby

Great Frigatebird

Golden Plover

Ruddy Turnstone

Wandering Tattler

Sanderling

Bristle-thighed Curlew

Sooty Tern

Gray-backed Tern

Brown-winged Tern

Common Noddy

Hawaiian Noddy

Blue-gray Noddy

Fairy Tern

Pomavice Jaeger $4+$ 
Location $18^{\circ} 05^{\prime} \mathrm{N} \times 160^{\circ} 20^{\prime} \mathrm{w}$ to $16^{\circ} 30^{\prime} \mathrm{N} \times 162015 \mathrm{~W}$

Weather $\quad$ Date 26 Jawuavy, 1965 Time 0630 to 1740

\section{$140 \mathrm{miles}$}

SPECIES

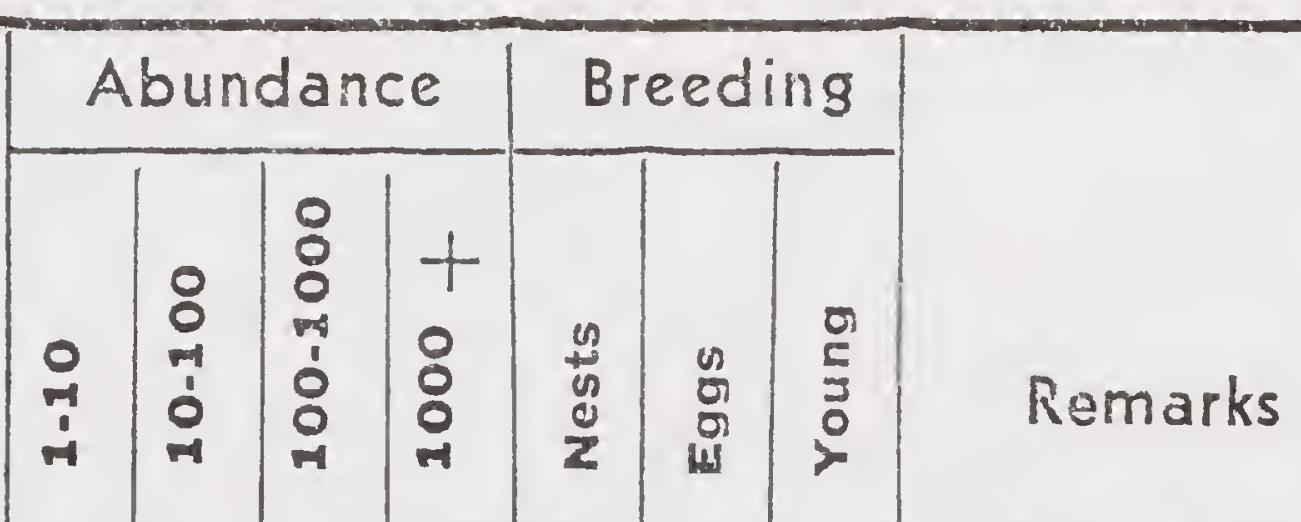

Laysan Albatross

Black-footed Albatross

Wedge-tailed Shearwater

Christmas 1. Shearwater

Audubon's Shearwater

Bonin 1. Petrel

Phoenix 1. Petrel

Bulwer's Petrel

Sooty Petrel

Red-tailed Tropicbird

White-tailed Tropicbird

Masked Booby

Brown Booby

Red-footed Booby

Great Frigatebird

Golden Plover

Ruddy Turnstone

Wandering Tattler

Sanderling

Bristle-thighed Curlew

Sooty Tern

Gray-backed Tern

Brown-winged Tern

Common Noddy

Hawaiian Noddy

Blue-gray Noddy

Fairy Tern

Leach's Stromplebed I

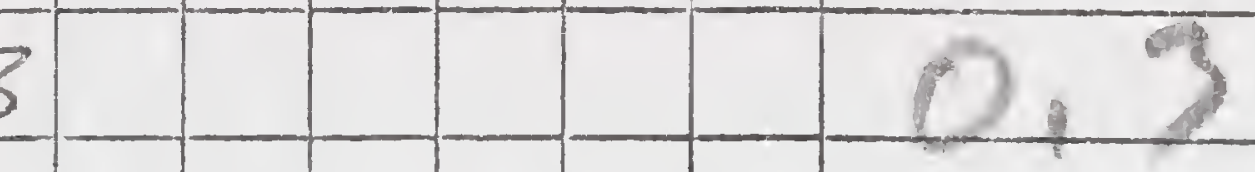

\section{/}

9 


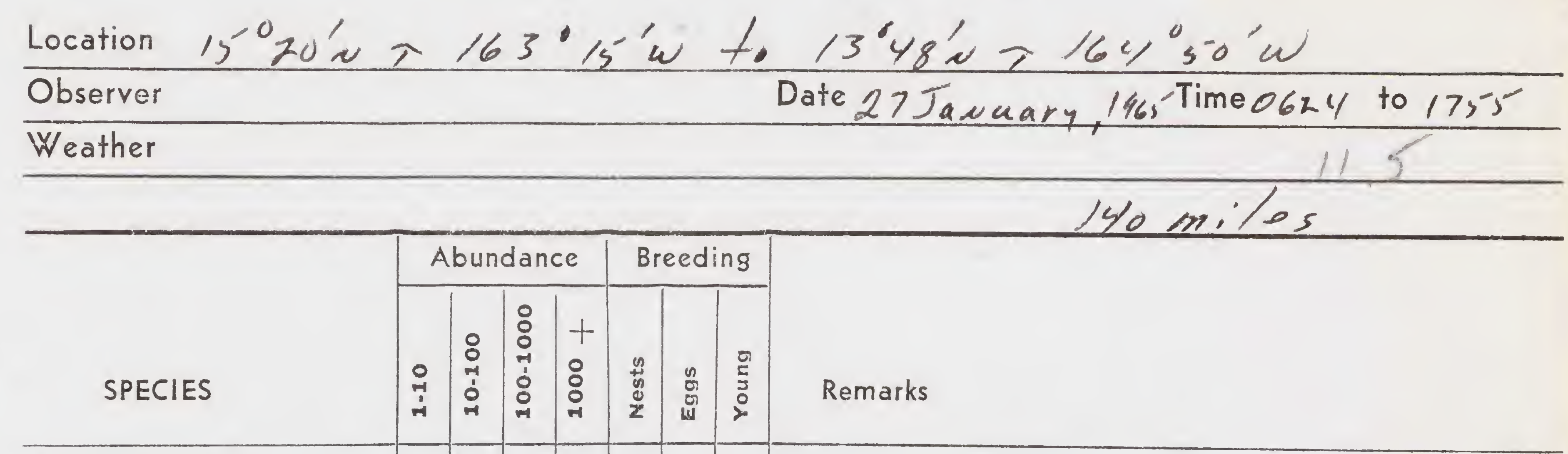

Laysan Albatross

Black-footed Albatross

Wedge-tailed Shearwater

Christmas I. Shearwater

Audubon's Shearwater

Bonin 1. Petrel

Phoenix 1. Petrel

Bulwer's Petrel

Sooty Petrel

Red-tailed Tropicbird

White-tailed Tropicbird

Masked Booby

Brown Booby

Red-footed Booby

Great Frigatebird

Golden Plover

Ruddy Turnstone

Wandering Tattler

Sanderling

Bristle-thighed Curlew

Sooty Tern

Gray-backed Tern

Brown-winged Tern

Common Noddy

2

Hawaiian Noddy

Blue-gray Noddy

Fairy Tern

Tuam Ferwande, petrs

$\frac{1}{2}$

D. 0

$$
\text { - }
$$

$19-\square-1.6$

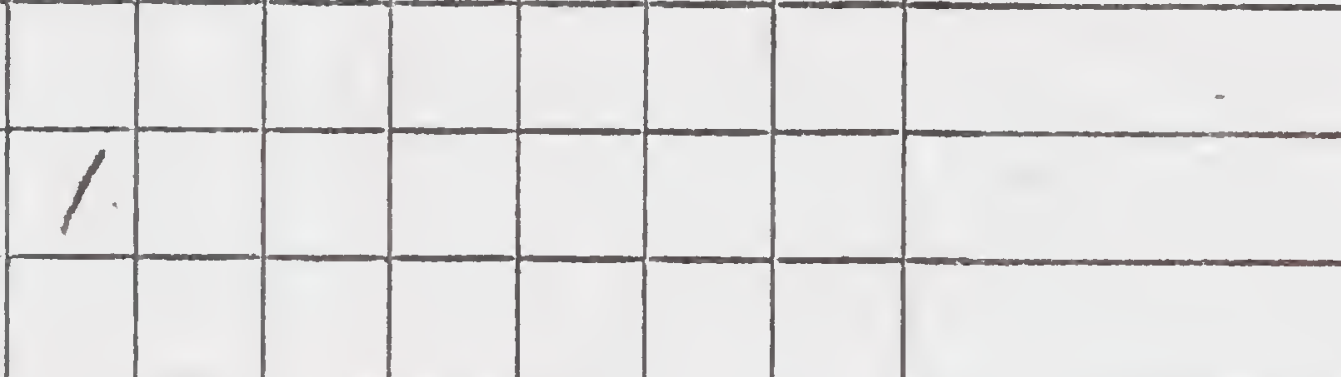

\begin{tabular}{l|l}
\hline Frigatebird & 2 \\
\hline pterdedrona & 1 \\
\hline Shearwator-Petrel & 2
\end{tabular} 
Location $12^{\circ} 05^{\prime} \sim>166^{\circ} \% 0^{\prime} \mathrm{w}$ to $10^{\circ} 5^{\circ} 0^{\prime} \approx>16>{ }^{\circ} 25^{\prime} \omega$

Weather

SPECIES

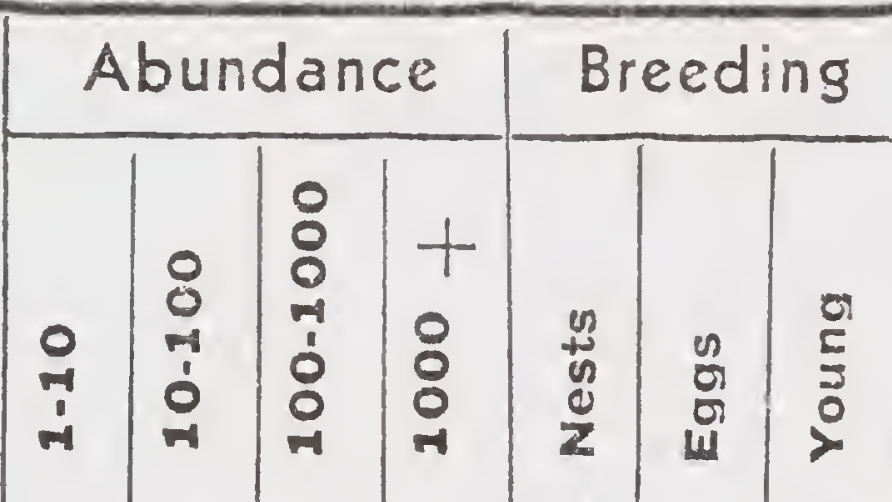

Remarks

Laysan Albatross

Black-footed Albatross

Wedge-tailed Shearwater

Christmas I. Shearwater

Audubon's Shearwater

Bonin 1. Petrel

Phoenix 1. Petrel

Bulwer's Petrel

Sooty Petrel

Red-tailed Tropicbird

White-tailed Tropicbird

Masked Booby

Brown Booby

Red-footed Booby

Great Frigatebird

Golden Plover

Ruddy Turnstone

Wandering Tattler

Sanderling

Bristle-thighed Curlew

Sooty Tern

Gray-backed Tern

Brown-winged Tern

Common Noddy

Hawaiian Noddy

Blue-gray Noddy

Fairy Tern

Jum Fervandey netred 4

Leach's Stom Peted 2 


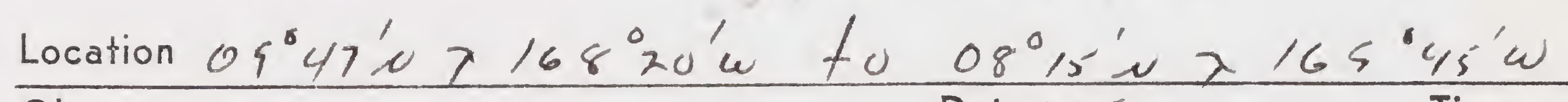

Weather $\quad$ Date29Jamuarm, 1965 Time 0633 to 1828 $130 \mathrm{miles}$

\begin{tabular}{|c|c|c|c|c|c|}
\hline Abun & danc & & & eed & \\
\hline $\begin{array}{l}8 \\
0 \\
1 \\
0 \\
0\end{array}$ & $\mid$\begin{tabular}{l}
0 \\
\multirow{1}{1}{} \\
$\dot{0}$ \\
0
\end{tabular} & $\begin{array}{l}T \\
\vdots \\
\vdots\end{array}$ & $\begin{array}{l}\frac{\tilde{y}}{\mathbf{y}} \\
\mathbf{z}\end{array}$ & : & $\begin{array}{l}\frac{0}{5} \\
\stackrel{0}{0}\end{array}$ \\
\hline
\end{tabular}

Laysan Albatross

Black-footed Albatross

Wedge-tailed Shearwater

Christmas 1. Shearwater

Audubon's Shearwater

Bonin 1. Petrel

Phoenix I. Petrel

Bulwer's Petrel

Sooty Petrel

Red-tailed Tropicbird

White-tailed Tropicbird

Masked Booby

Brown Booby

Red-footed Booby

Great Frigatebird

Golden Plover

Ruddy Turnstone

Wandering Tattler

Sanderling

Bristle-thighed Curlew

Sooty Tern

Gray-backed Tern

Brown-winged Tern

Common Noddy

Hawaiian Noddy

Blue-gray Noddy

Fairy Tern

Black Growded Albatross

Juan Zerwundey, Patrel

Kermadec petrel

No vilen a cole plara

Friate bind

tern

pterodroma

Shearwuter-petvel

$\frac{1}{1}$

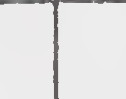

(3) 


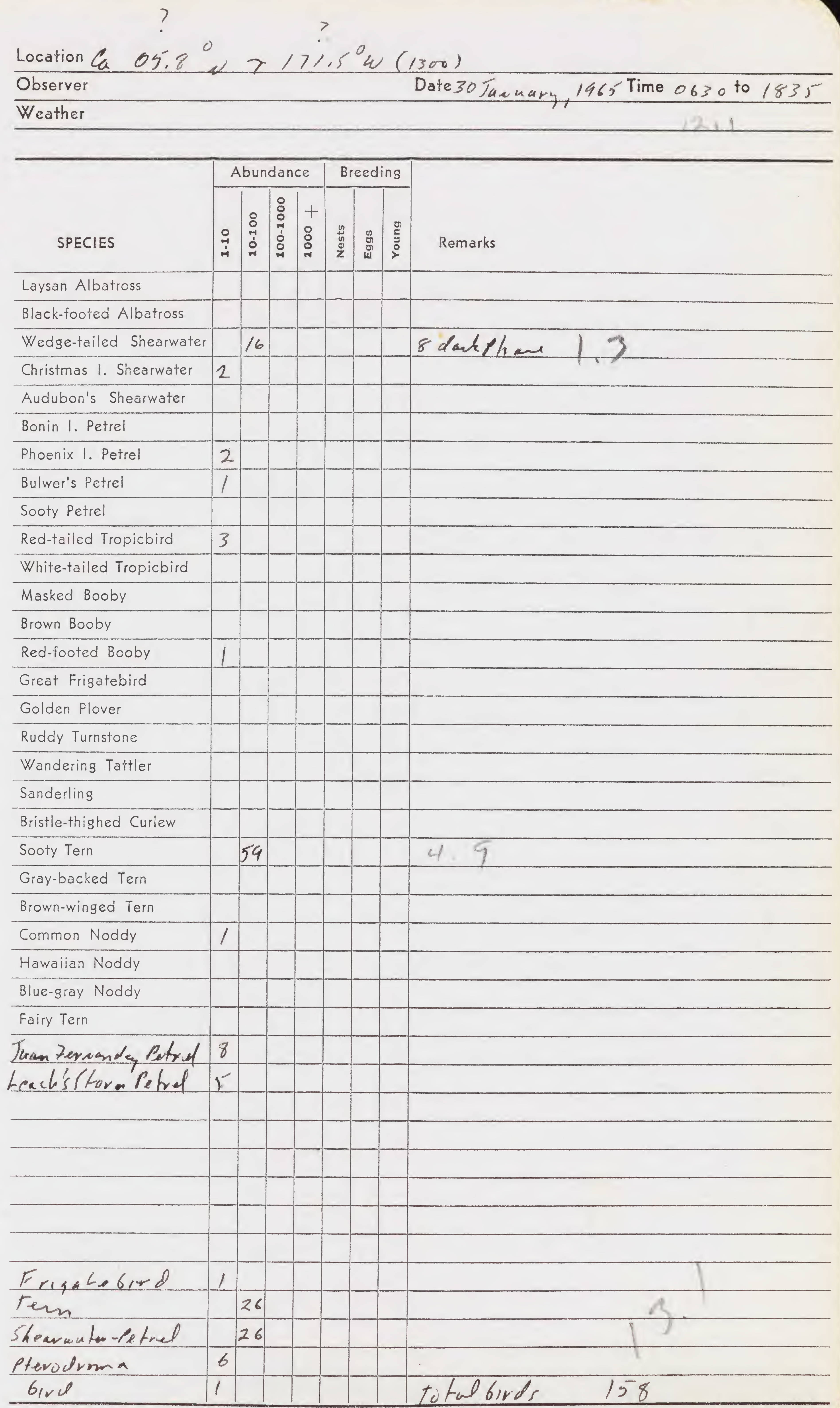




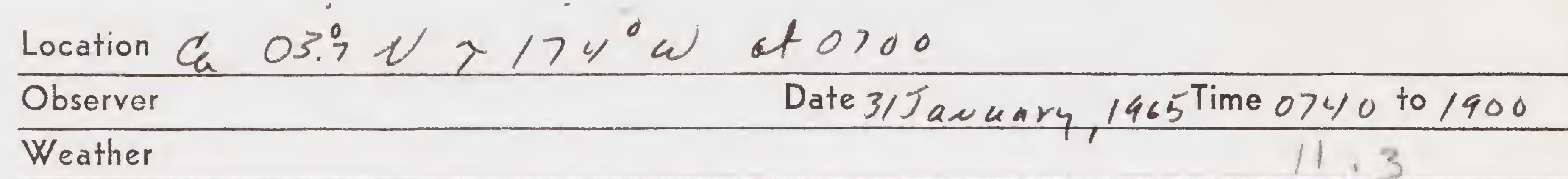

\begin{tabular}{|c|c|c|c|c|c|c|c|}
\hline \multirow[b]{2}{*}{ SPECIES } & \multicolumn{3}{|c|}{ Abundance } & \multicolumn{3}{|c|}{ Breeding } & \multirow[b]{2}{*}{ Remarks } \\
\hline & $\underset{7}{Z}$ & $\left|\begin{array}{l}0 \\
0 \\
1 \\
0 \\
0\end{array}\right|$ & $\begin{array}{ll}0 \\
0 \\
0 \\
0\end{array}$ & 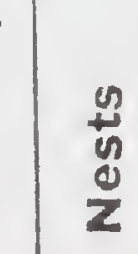 & 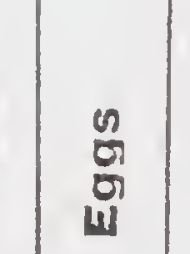 & $\begin{array}{l}0 \\
\end{array}$ & \\
\hline
\end{tabular}

Laysan Albatross

Black-footed Albatross

Wedge-tailed Shearwater 7

Christmas 1. Shearwater

Audubon's Shearwater /

Bonin 1. Petrel

Phoenix 1. Petrel

Bulwer's Petrel

3 dact shar O 6

Sooty Petrel

Red-tailed Tropicbird

White-tailed Tropicbird

Masked Booby

Brown Booby

Red-footed Booby

Great Frigatebird

Golden Plover

Ruddy Turnstone

Wandering Tattler

Sanderling

Bristle-thighed Curlew

Sooty Tern

Gray-backed Tern

Brown-winged Tern

Common Noddy

Hawaiian Noddy

Blue-gray Noddy

Fairy Tern

Leach's Storm Petrel

Juar Fevwandey Petcel

$185^{-} \quad$ many bmomatmes 16,4

\begin{tabular}{l|l|l|l}
\hline Frisatebird & 3 & & \\
\hline Tema & & & 310 \\
\hline $6006 y$ & 1 & & \\
\hline Stormpetred & 1 & & \\
\hline Shearwater-Petrel & & 10 &
\end{tabular} 


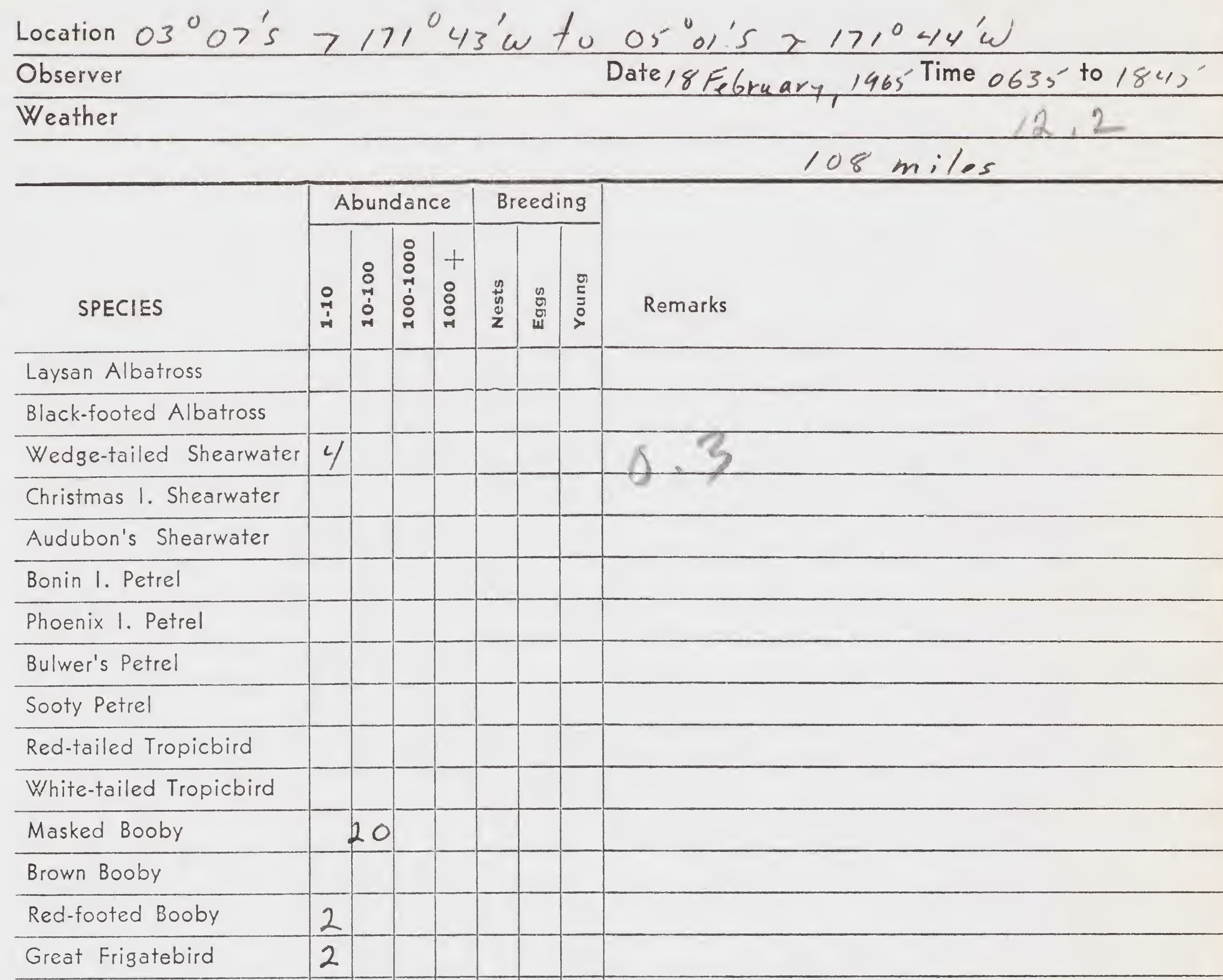

Golden Plover

Ruddy Turnstone

Wandering Tattler

Sanderling

Bristle-thighed Curlew

\begin{tabular}{l|l|l|l|}
\hline Sooty Tern & 89 & 73,0 \\
\hline Gray-backed Tern & 26 & &
\end{tabular}

Brown-winged Tern

Common Noddy

Hawaiian Noddy

Blue-gray Noddy

Fairy Tern

Lesserfricatebid 2

Leach's Stormbtrel 5

Frigate6r-d

teve

Sheavater-Retrel

ptevodrom a 
Location $06^{\circ} 41^{\prime} 57171^{\circ} 30^{\prime} w$ fo $09^{\circ} 13^{\prime} 5 \times 171^{\circ} 04^{\prime} \omega$ Observer Date/9February, 1965 Time 0630 to 1845

Weather 12,3 127 miles

SPECIES

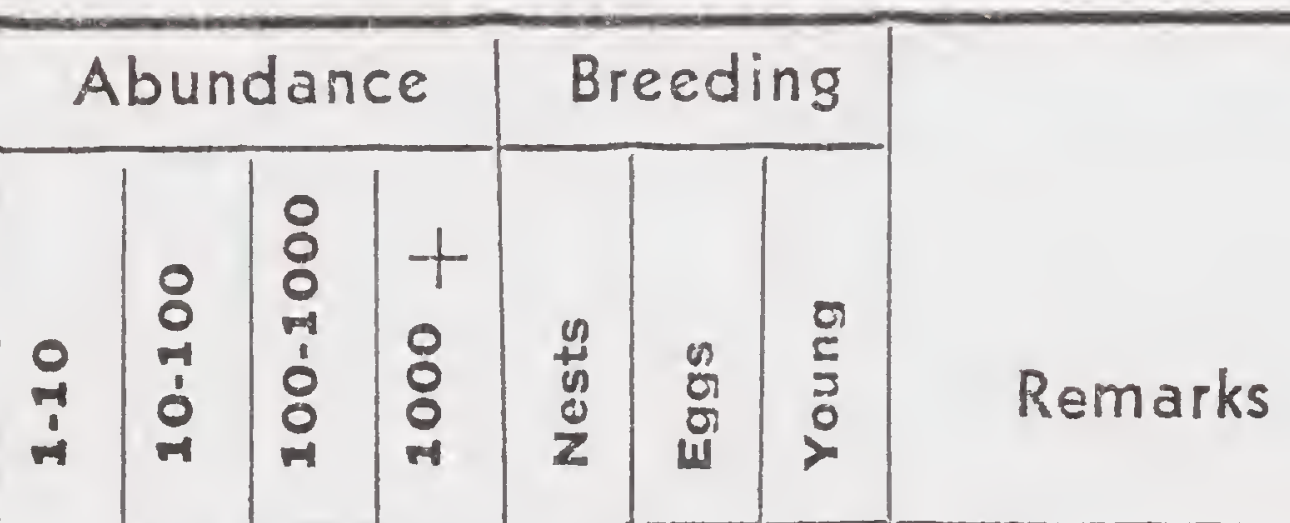

Laysan Albatross

Black-footed Albatross

Wedge-tailed Shearwater

Christmas 1. Shearwater

Audubon's Shearwater

Bonin I. Petrel

Phoenix 1. Petrel

Bulwer's Petrel

Sooty Petrel

Red-tailed Tropicbird

White-tailed Tropicbird

Masked Booby

Brown Booby

Red-footed Booby

Great Frigatebird

Golden Plover

Ruddy Turnstone

Wandering Tattler

Sanderling

Bristle-thighed Curlew

Sooty Tern

Gray-backed Tern

Brown-winged Tern

Common Noddy

Hawaiian Noddy

Blue-gray Noddy

Fairy Tern

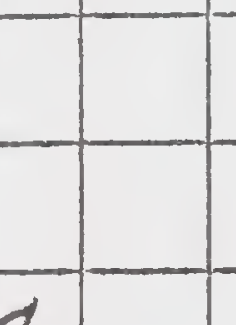

ceaclísfor Petrel

\section{7}

40

3,3

Frigate 6ird

Shearwater-letrel

Pterdroma

$6,-8$ 
Location $10^{\circ} \mathrm{c} / 6^{\prime}>170^{\circ} 49^{\prime} w$ to $12^{\circ} 36^{\prime} 5>170^{\circ} 40^{\prime} w$

\begin{tabular}{|c|c|c|c|c|c|c|c|}
\hline Observer & & & & & & & Datezo FeGruavy 1965 Time 0630 to, 845 \\
\hline Weather & & & & & & & 12,3 \\
\hline & & & & & & & 106 n:105 \\
\hline & & ind & & & eedi & & \\
\hline SPECIES & $\stackrel{1}{1}$ & 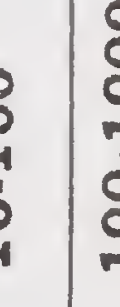 & $\begin{array}{l}+ \\
\vdots \\
\vdots\end{array}$ & \begin{tabular}{|l}
0 \\
0 \\
0 \\
0 \\
$z$ \\
$z$
\end{tabular} & $\mid \begin{array}{l}n \\
0 \\
5 \\
w \\
w\end{array}$ & 是 & Remarks \\
\hline
\end{tabular}

Laysan Albatross

Black-footed Albatross

Wedge-tailed Shearwater

Christmas I. Shearwater

16

Audubon's Shearwater

Bonin 1. Petrel

Phoenix 1. Petrel

Bulwer's Petrel

Sooty Petrel

Red-tailed Tropicbird

White-tailed Tropicbird

1

Masked Booby

Brown Booby

Red-footed Booby

Great Frigatebird

Golden Plover

Ruddy Turnstone

Wandering Tattler

Sanderling

Bristle-thighed Curiew

Sooty Tern

Gray-backed Tern

Brown-winged Tern

Common Noddy

Hawaiian Noddy

Blue-gray Noddy

Fairy Tern

9

80

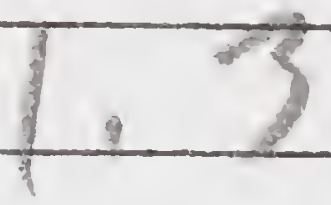

Sheowater-Petred

Gird 
Location

Observer

Date 257elruary, 1965 Time/000 to 16/2

Weather

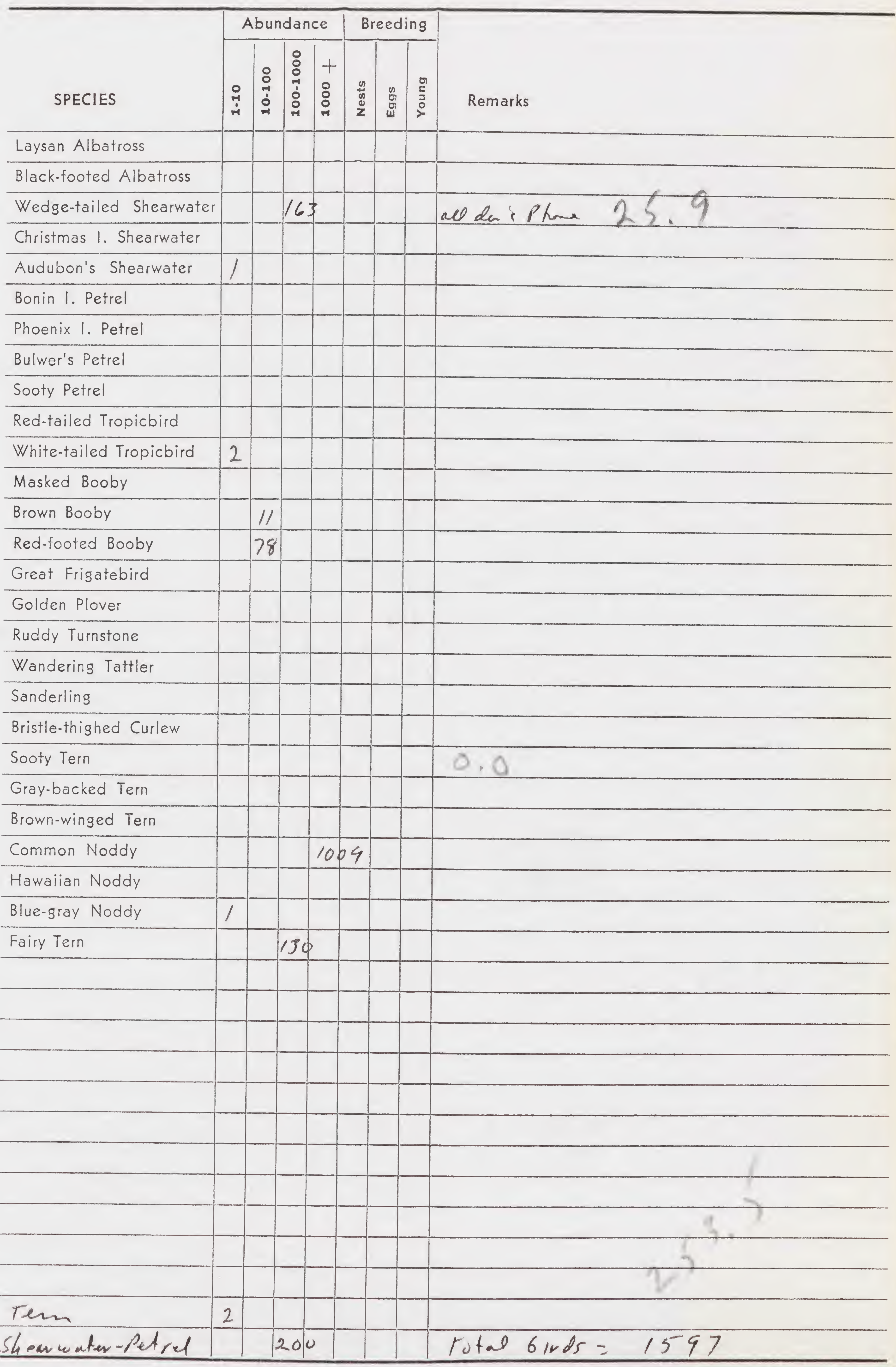

